Review Article

\title{
Effectiveness of the Laser Application in Temporomandibular Joint Disorder: A Systematic Review of 1172 Patients
}

\author{
Abdalwhab Zwiri ${ }^{10},{ }^{1}$ Manal Ahmad Alrawashdeh, ${ }^{2}$ Mohammad Khan, ${ }^{1}$ \\ Wan Muhamad Amir W. Ahmad, ${ }^{1}$ Nur Karyatee Kassim,, ${ }^{1,3}$ Jawaad Ahmed Asif,,3 \\ Khoo Suan Phaik, ${ }^{4}$ Adam Husein, ${ }^{1,3}$ and Zuryati Ab-Ghani $\mathbb{D}^{1,3}$ \\ ${ }^{1}$ School of Dental Sciences, Universiti Sains Malaysia, Health Campus, 16150 Kubang Kerian, Kota Bharu, Kelantan, Malaysia \\ ${ }^{2}$ School of Computer Sciences, Universiti Sains Malaysia, 11800 Gelugor, Penang, Malaysia \\ ${ }^{3}$ Hospital Universiti Sains Malaysia, 16150 Kubang Kerian, Kota Bharu, Kelantan, Malaysia \\ ${ }^{4}$ Department of Oral Diagnostic and Surgical Sciences, School of Dentistry, International Medical University, \\ 57000 Bukit Jalil, Kuala Lumpur, Malaysia
}

Correspondence should be addressed to Abdalwhab Zwiri; dr_zwiri@yahoo.com and Zuryati Ab-Ghani; zuryati@usm.my

Received 1 May 2020; Accepted 24 August 2020; Published 12 September 2020

Academic Editor: Mieszko Wieckiewicz

Copyright (c) 2020 Abdalwhab Zwiri et al. This is an open access article distributed under the Creative Commons Attribution License, which permits unrestricted use, distribution, and reproduction in any medium, provided the original work is properly cited.

\begin{abstract}
Objective. The aim of this systematic review was to evaluate the effectiveness of laser application in temporomandibular joint disorder. Methods. PubMed, SCOPUS, Science Direct, Web of Science, and Google Scholar electronic databases were searched systematically with restricting the languages to only English and year (January 2001 to March 2020), and studies were selected based on the inclusion criteria. Study quality and publication bias were assessed by using the Robvis, a software package of $R$ statistical software. Results. This systematic review included 32 studies (1172 patients) based on the inclusion and exclusion criteria. Most of the studies reported significant reduction of pain by the use of the laser during TMD treatment. Two-thirds of the study (78.13\%) found a better outcome comparing with conventional one. According to Robvis, $84.4 \%$ of the studies were high methodological studies with low risk of bias. Conclusion. TMD patients suffer with continuous pain for long time even after conventional treatment. Laser therapy shows a promising outcome of pain reduction for TMD patients. Therefore, laser therapy can be recommended for the TMD patients' better outcome. This trial is registered with PROSPERO (CRD42020177562).
\end{abstract}

\section{Introduction}

Temporomandibular disorder (TMD) is defined as a series of clinical problems involving muscles of mastication, temporomandibular joints (TMJ), and related structures, identified by facial pain in the TMJ region and masticatory muscle, limited or deviated mandibular movement, and TMJ sounds during jaw movement and action [1]. While they have long been the subject of research, there are still many questions regarding their etiology, diagnosis, and management. Multifactorial TMD etiology is widely established, comprising the involvement of parafunctional behaviors, trauma, stress, and psychological, systemic, genetic, and occlusal causes. None of these variables has proved to outweigh the others, however [2]. The main reason for pain in the orofacial area that does not derive from dental arches is the TMD. In the community, at least one sign is confirmed by $40 \%-75 \%$ of healthy individuals, and at least one symptom of TMD is observed by $33 \%$. $40-75 \%$ of healthy individuals in the population have at least one TMD sign, and 33\% have at least one TMD symptom. Periarticular tissues (capsule, synovium, and TMJ ligaments), collateral ligaments, and posterior attachments are the most affected anatomical structures of TMJ due to these diseases [3]. Depending on the multifactorial etiology of these problems, the treatment typically requires more than one approach to optimize any potential results, such as medication, behavioral therapy, and physical therapy [2]. 


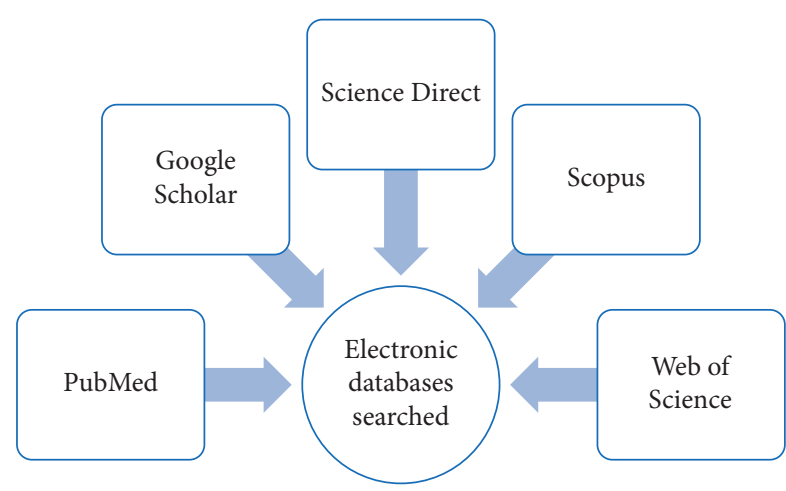

FIgURE 1: Five electronic databases searched for this review.

In the last few years, laser light has been extensively used in clinical dentistry for the treatment of soft tissue disorders, hypersensitivity of dentine, bone regeneration, and musculoskeletal pain. In TMD patients, LLLT has been used by conservative treatment methods to enhance function and decrease symptoms [3]. The main impacts of laser (LLLT) are biosimulative, regenerative, analgesic, and anti-inflammatory [4]. Helium-neon laser (He-Ne gas) and infrared laser with gallium-arsenium (Ga-As) diode or gallium-aluminum-arsenium (Ga-Al-As) rays are the most common types of laser rays. [5]. The LLLT, known as a soft laser, has low energy intensity and has no effect on skin temperature. The main effect of LLLT is based on the light-absorption process. This soft laser has a wavelength of between $630 \mathrm{~nm}$ and $1300 \mathrm{~nm}$ [3]. The relative clinical effectiveness of LLLT in treating temporomandibular (TMD) disorders is controversial. Several authors identified LLLT's effectiveness as superior to placebo treatment, while others observed no significant differences between LLLT and placebo for TMJ pain measures, in the view of fact that outcomes in LLLT trials can rely on sample size, population, treatment protocols, and methodology $[6,7]$.

Therefore, the aim of this systematic review was to find out the effectiveness of laser application in temporomandibular joint pain and review the evidence from previous studies with their sample size and methodology in the management of TMD. This review will provide a precise and obvious knowledge about the benefits and procedures of laser application, which have already been successfully established in TMD management.

\section{Materials and Methods}

2.1. Search Strategy. Articles were searched in five electronic databases (Figure 1) where the following keyword combinations were used: TMJD + laser, TMJ problem + laser, TMD + laser, TMJD/TMD management + laser application, and TMJ + laser application. Articles between the year 2001 and 2020 were reviewed and systematically searched for those literature published until March 2020. After final screening, total of 32 articles were included in this systematic review. The search encompasses articles (full text) written in English and published in peer-reviewed journals related to TMJ diseases where laser application was performed in different levels.
2.2. Study Selection. Here, the prime concern was to find out the uses of laser in temporomandibular disorder patients in terms of reduction of pain and tenderness, improvement of mouth opening and joint sounds, and improvement in the range of jaw motion. The criteria for inclusion have been established as papers using the search keywords mainly TMJ problems and laser application. At the other side, the papers that use laser in TMJ diseases along with other problems such as anatomical defects, anomalies, myofascial pain disorder syndrome (MPDS), soft and hard tissue pathology (tumor, cancer), and previous record of TMJ surgery were excluded from the study. In the context of the exclusion criteria, it also added that those studies were not conducted in human (such as animal studies), and publications in other languages beside English were excluded. The case reports and letter to editor were also excluded from this review. Titles and abstracts of identified studies were assessed independently to judge if the studies match the inclusion criteria.

2.3. Data Extraction and Organization. Data were extracted based on the first author, year of publication, number of samples, age and gender of samples, types of TMJ problem, laser types, laser energy and application rate, and results. The data were extracted and double-checked by the authors.

\section{Results}

3.1. Selection of Studies. At the beginning, this research search strategy provided a total of 5889 papers from databases such as PubMed, Web of Science, Google Scholar, SCOPUS, and ScienceDirect. The remaining 2378 papers were further screened after eliminating 3511 papers in the detection phase (nonhuman topics, summary documents, case reports, editorials, letters and comments, and duplicate studies). A total of 72 studies were considered worthy, but due to unusable data format, forty studies were excluded. Thus, eventually, based on the research goals and inclusion and exclusion requirements, 32 studies (1172 TMD patients in total) were included in this study (Figure 2), and the full text of all the included studies has been retrieved.

3.2. Study Characteristics. The key characteristics of the included studies are presented in Table 1. All the studies included were journal articles and most are adults. Among these 32 studies, thirteen were conducted in Brazil, five in European continent and Iran, respectively, three from India, two from Turkey and Taiwan, and one from Malaysia and Iraq. Ga-Al-As (LLLT) laser with a variation of 780-904 nm wavelength is used in most of the studies to treat the TMD patients. Twenty-five studies reported better outcome by reduction of TMD pain compared with conventional treatment modalities, while 7 studies did not find any significant difference between conventional and laser treatment. 


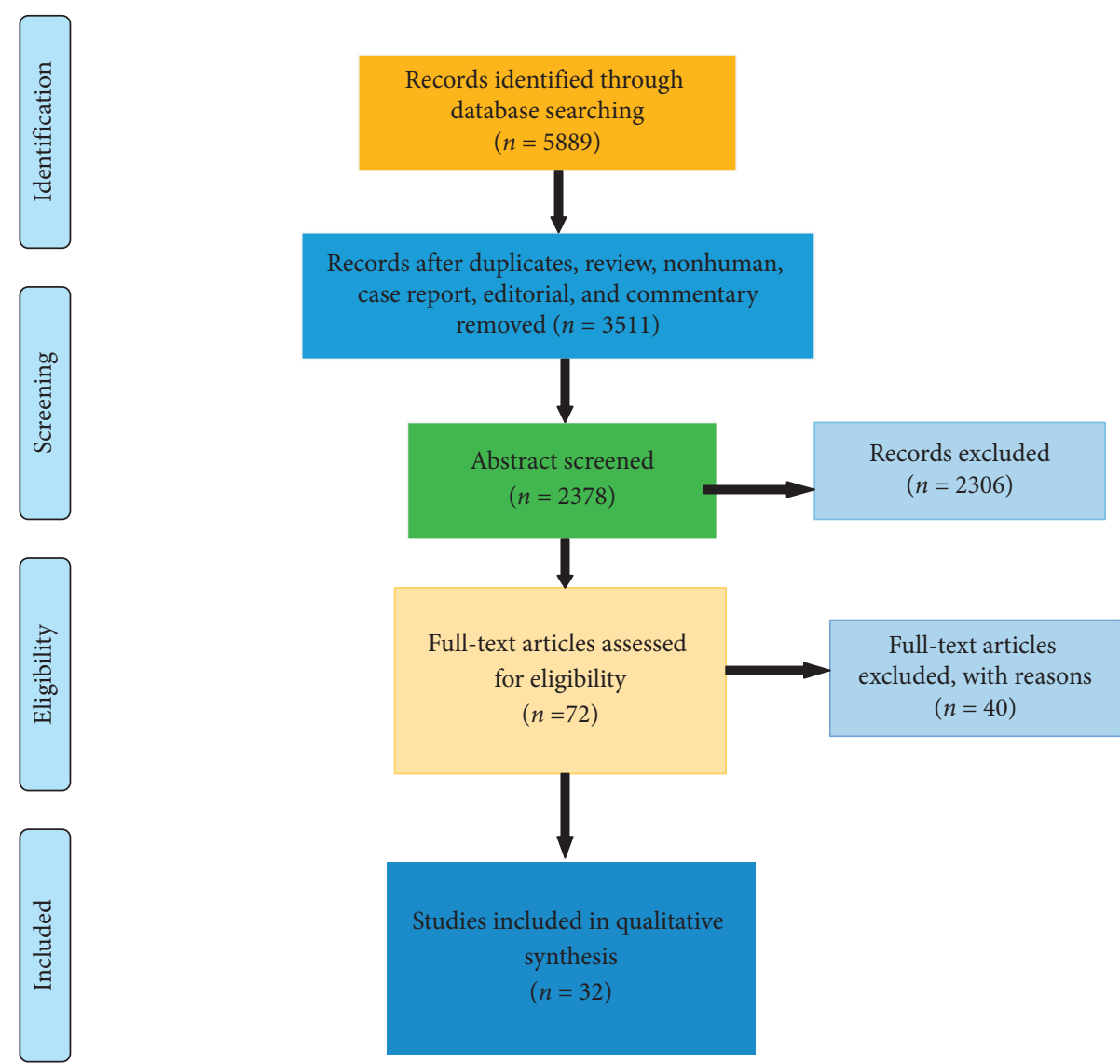

FIgURE 2: PRISMA flow chart diagram of search strategy and selection of the studies.

3.3. Risk of Bias Assessment. Publication bias was assessed by using $R$-based Robvis software package introduced by the National Institute for Health Research (NIHR) [36]. Based on visual inspection of the figure, there is no protentional publication bias in this study assessing the effectiveness of laser treatment for TMD patients (Figure 3). Out of 32 studies, twenty-seven (84.4\%) are high methodological studies, which have an overall low risk of bias with some concerns, while only 5 studies have only high risk of bias.

\section{Discussion}

TMD management is very complex and contentious due to the difficulty of determining the exact reasons of the disease and its multifactorial character. The severity varies greatly, and the procedure is varying in terms of duration and invasiveness. Nevertheless, TMD treatment is intended to minimize discomfort, enhance mobility, and delay the progression of internal derangement as accepted by the American Society of Temporomandibular Joint Surgeons guidelines [1]. TMD has a multifactorial nature or etiology; therefore, it is very difficult to get the desired treatment outcome, especially for those patients experiencing severe pain and limited jaw movements.

However, this systematic review tried to overview the role of laser in the management of TMJ disorder patients. After completing this review, the result showed a huge role of laser in TMD management. Most of the studies used LLLT for management of TMD, where it showed a tremendous action in reducing pain, joint clicking, muscle tenderness, and jaw movements.

A study was conducted by Kulekcioglu et al. in 2003 among Turkish population to investigate the effectiveness of low-level laser therapy in the treatment of TMD. Results of the study showed a significant reduction of pain and improvement in maximum mouth opening, lateral motion, and number of tenderness points. According to Kulekcioglu et al. , LLLT in treating TMD may be considered as an alternative physical modality [8]. Another study by Kogawa et al. also found an increase in maximum mouth opening and a decrease in tenderness to palpation in TMD patient after receiving LLLT. Author recommended that LLLT was effective in the management of myogenic TMD [9].

On the other hand, some researchers also tried to find out the effectiveness of the laser treatment in TMD patient, and they concluded with no significant role of LLLT. In 2005, Abreu et al. conducted a study to assess the efficacy of low-intensity laser therapy (LILT) in temporomandibular joint (TMJ) pain and mandibular dysfunction patients. The study had 2 groups, placebo and experimental (LILT). They used the infrared laser $(780 \mathrm{~nm}, 30 \mathrm{~mW}, 10 \mathrm{~s}$, and $6.3 \mathrm{~J} / \mathrm{cm} 2)$ at three TMJ points. Even though the patient treated with laser had good pain reduction, the result showed no significant changes between placebo and laser groups. 


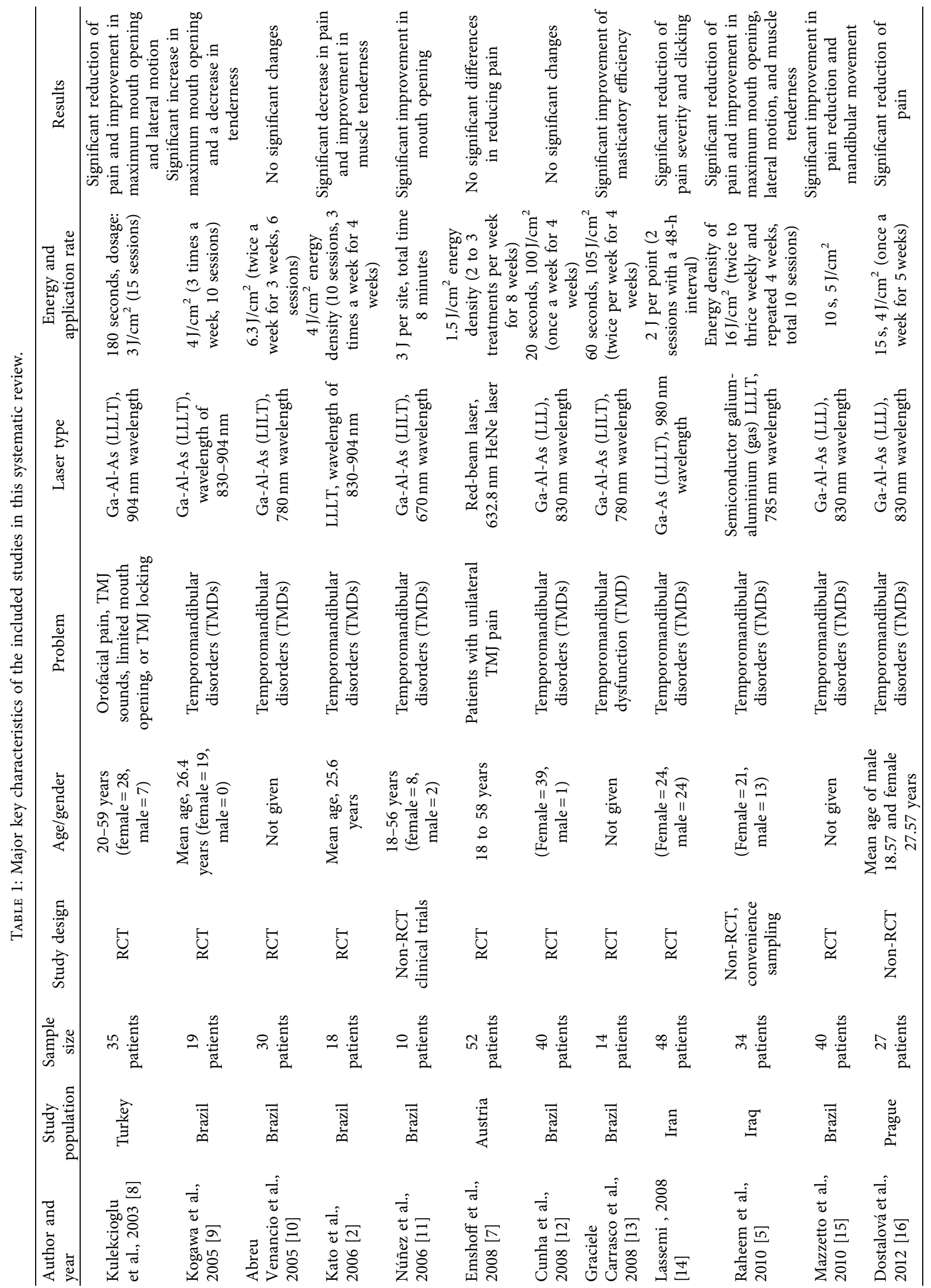




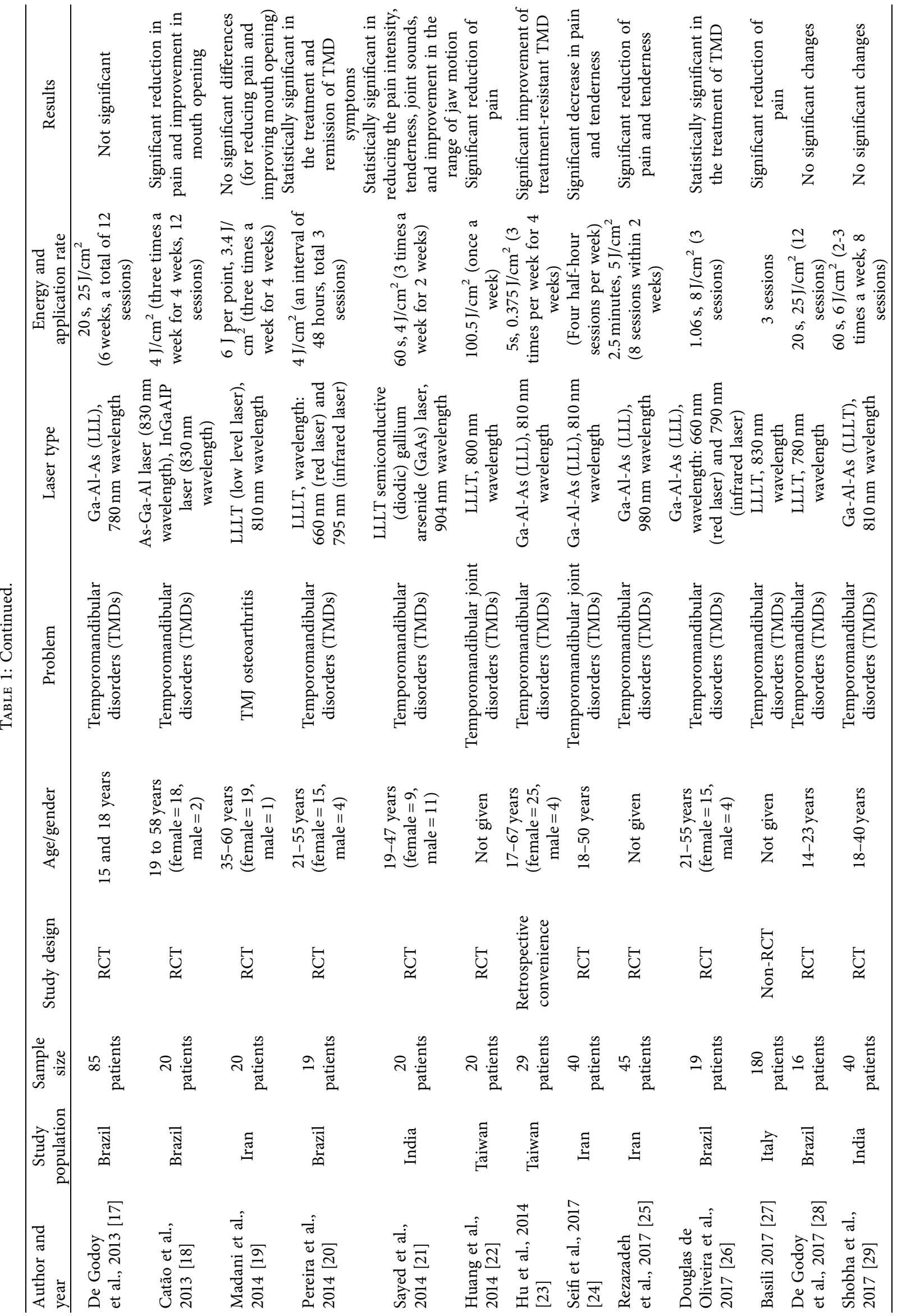




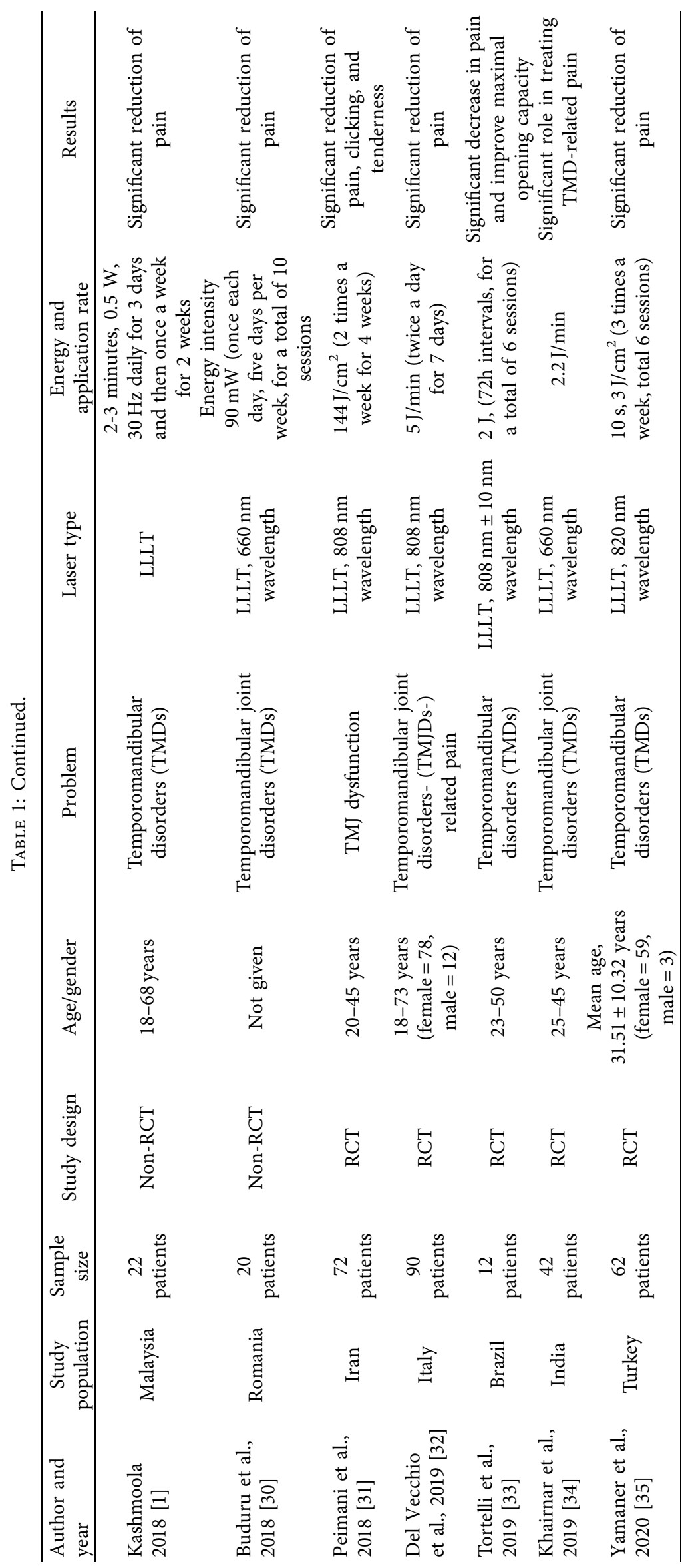




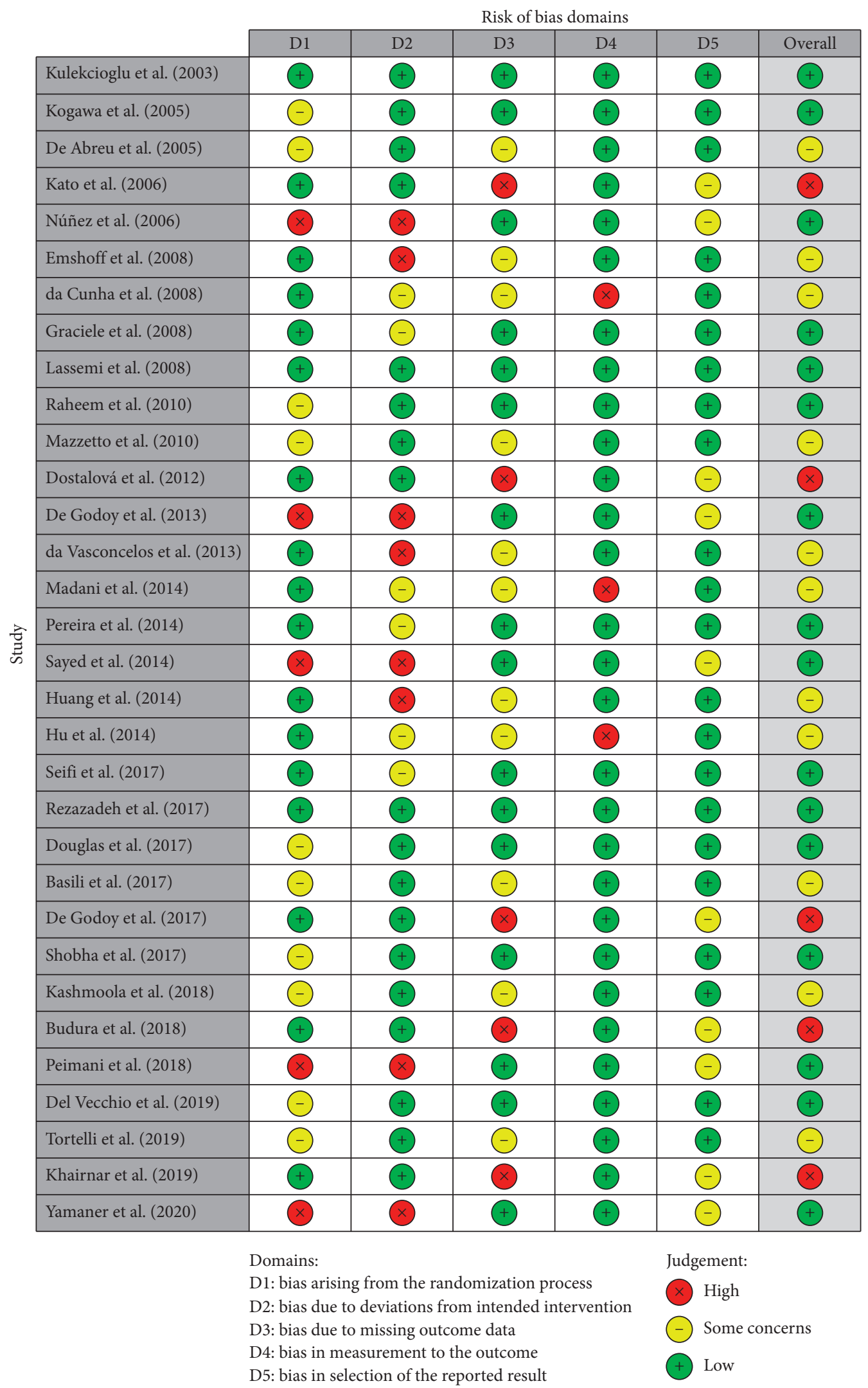

Figure 3: Risk of bias assessment of the study. 
Therefore, the researcher did not recommend infrared LILT as a better treatment option. Though there are benefits of applying laser in TMDs management because of noninvasiveness and cost efficient, it has no reported side effects [10]. Another research was performed by Emshoff et al. in Austrian population to evaluate the effectiveness of LLLT in TMJ pain management. A red-beam laser (Model 2000; Helbo Medizintechnik, Austria) (632.8 nm HeNe laser, continuous wave, $30 \mathrm{~mW}$ output power, $1.5 \mathrm{~J} / \mathrm{cm} 2$ energy density) was used. They used three follow-ups from baseline to measuring the visual analogue scale (VAS). The results also presented no significant changes in the management of TMJ. The study recommends that LLLT is no better at minimizing TMJ pain during action than placebo [7]. Similarly, da Cunha et al. reported no significant difference between placebo and laser groups [12]. Authors used Ga-AlAs (gallium-aluminium-arsenide) low-level laser (Biolux laser - Bio-Art, São) with $830 \mathrm{~nm}$ wavelength and an output of $500 \mathrm{~mW}$ for $20 \mathrm{sec}$. This study used craniomandibular index (CMI) and VAS for measuring effectiveness of treatment, which results in no significant difference in 2 different protocols, while the patient treated with LLLT reported better pain reduction [12].

Although some authors did not notice any important differences, some studies showed better results when comparing the LLLT with a placebo control group. In 2010, Raheem et al. observed that LLLT plays a significant role in TMDs management by reducing pain and improving maximum mouth opening, lateral motion, and muscle tenderness. Raheem et al. advised LLLT as an effective therapeutic option in myofascial pain dysfunction of TMJ for its analgesic and functional improvement [5].

Even though LLLT is a type of treatment widely applied in physiotherapy of musculoskeletal disorders, there are only some studies that discuss its use in the management of TMD. In 2012, Dostalová et al. performed a research to observe the activity of TMJ and its surrounding tissues and compared the objective results of the effect of LLLT. LLLT was beneficial in the progress of the range of TMD and facilitated a significant pain symptoms reduction [16]. According to Catao et al. , laser therapy was very effective in the pain control and mouth opening of TMDs patients [18]. A study conducted by Sayed et al. confirmed LLLT with satisfactory outcome reducing the pain intensity, number of tender points, joint sounds, and improvement in the range of jaw movement. Therefore, it is an effective and efficient method for treating TMDs [21].

Few more studies have been performed in 2017 by several researchers to evaluate the effectiveness of laser therapy in the treatment of TMDs. Based on the sample size, population, and study design, the result showed some controversy about laser treatment. In 2017, Rezazadeh et al. examined 45 Iran patients to discover the effectiveness of transcutaneous electrical nerve stimulation (TENS) and LLLT in treatment of TMD patients who did not respond to pharmacological therapy. The result showed a significant reduction of pain and tenderness in TMD patients [25]. Another study was performed by Seifi et al. in 40 patients of Iran to assess the result of low-level laser (LLL) therapy and transcutaneous electric nerve stimulation (TENS) on TMDs. The author suggested that TENS or LLL therapy may be useful in improving TMD symptoms at least for the short term [24]. de Godoy et al. carried out a study using a wavelength of $780 \mathrm{~nm}$, energy density of $25 \mathrm{~J} / \mathrm{cm}^{2}$, power of $50 \mathrm{~mW}$, power density of $1.25 \mathrm{~W} / \mathrm{cm}^{2}$, and a 20 -second exposure. He did not find any significant differences after laser application [28]. This may be the use of the measurement tool used in the study. They compared the difference with the help of electromyography (EMG) signal, while VAS is more specific, accurate, and widely used for pain assessment. Similarly, Shobha et al. conducted a study using 8 sessions of active LILT with a specific diode laser (gallium-aluminum-arsenide, $810 \mathrm{~nm}, 0.1 \mathrm{~W}$ ), while the most commonly used therapeutic laser in laser research has been the Ga-As-Al, a semiconductor laser. The laser group showed better improvement in pain reduction even after the 1-month follow-up compared to the placebo group in the VAS score, having no overall significant differences after receiving LLLT [29].

Though, clinically, the use of LLLT is a better procedure in managing TMJ pain. In 2018, a study completed by Buduru et al. showed a significant pain reduction and noticed that there is no disadvantage of LLLT. Thus, the author had recommended the use of LLLT for pain reduction in TMD patients [30]. According to Del Vecchio et al. , LLLT can significantly reduce TMD pain symptom, and it is very much effective in TMJD pain management (Del Vecchio et al. ). Another study performed by Khairnar et al. also found a significant reduction of TMDs pain with LLLT. That study recommends LLLT for treating TMD-related pain with no underlying bony pathology [34].

In the present year 2020, a study was conducted by Yamaner et al. in Turkey to investigate the impact of the ozone and low-level laser (LLL) therapies on pain and function in TMDs patients with disc displacement with reduction. The results of the study support the application of ozone as an effective therapeutic tool for pain relief and LLL as a supportive therapy for temporomandibular disorders [35]. In this systematic review, the author tried to investigate the effectiveness of laser application in temporomandibular joint pain. However, the goal of this systematic review has been achieved. From the above discussion, it is clear that the use of laser in TMD patient is controversial because of its positive and negative outcomes in several studies. But after this review, it can be clearly suggested that the use of the laser has been recommended by most of the researchers. Laser application plays an effective and potential role in the treatment of TMDs patients.

Although the present study went through a systematic search strategy and review of the selective articles, one of the limitations of the present study was the database searching. Due to the limited access of database, the author only searched in five specific databases. This study advised to perform another systematic review with meta-analysis by including some more databases searching to strengthen the findings. 


\section{Conclusion}

TMD patients mostly suffer with pain symptoms along with other problems. Nowadays, LLLT became very popular because of its effective role in pain reduction and no known side effects. This systematic review evaluated the effectiveness of the laser application in TMD patient by thorough investigation of the previous studies that have been conducted on laser. After this systematic review, LLLT can be recommended as a beneficial treatment approach for TMD patients.

\section{Conflicts of Interest}

The authors declare that they have no conflicts of interest.

\section{Authors' Contributions}

All authors listed have made a substantial, direct, and intellectual contribution to the work and approved it for publication. All authors were involved in this study in different phases. AZ, MAA, and MK were involved in the study protocol design and database searching. ZG, AZ, and MK were involved in the study selection. Any dispute was discussed and solved with WMWA, NKK, JAA, KSP, and AH. AZ and MK wrote the manuscript, and all others edited and improved the manuscript for submission standard.

\section{Acknowledgments}

The authors would like to acknowledge USM fellowship.

\section{References}

[1] M. A. Kashmoola, "A pilot study on the use of low level laser therapy in treatment of temporomandibular disorder," Journal of International Dental and Medical Research, vol. 11, no. 2, pp. 669-675, 2018.

[2] M. T. Kato, E. M. Kogawa, C. N. Santos, and P. C. R. Conti, "TENS and low-level laser therapy in the management of temporomandibular disorders," Journal of Applied Oral Science, vol. 14, no. 2, pp. 130-135, 2006.

[3] S. Ayyildiz, F. Emir, and C. Sahin, "Evaluation of low-level laser therapy in TMD patients," Case Reports in Dentistry, vol. 2015, pp. 1-5, Article ID 424213, 2015.

[4] D. Shukla and M. Muthusekhar, "Efficacy of low-level laser therapy in temporomandibular disorders: A systematic review," National Journal of Maxillofacial Surgery, vol. 7, no. 1, p. 62, 2016.

[5] S. M. A. Raheem, K. J. Hanau, and H. I. Abdulla, "The effects of low-level laser therapy and microcurrent electrical neural stimulation in the management of myofascial pain dysfunction of temporomandibular joint (a clinical comparative study)," Journal of Baghdad College of Dentistry, vol. 22, no. 2, pp. 45-52, 2010.

[6] J. Herranz-Aparicio, E. Vazquez-Delgado, J. Arnabat-Dominguez, A. Espana-Tost, and C. Gay-Escoda, "The use of low level laser therapy in the treatment of temporomandibular joint disorders. Review of the literature," Medicina Oral Patología Oral Y Cirugia Bucal, vol. 18, no. 4, pp. e603-e612, 2013.
[7] R. Emshoff, R. Bösch, E. Pümpel, H. Schöning, and H. Strobl, "Low-level laser therapy for treatment of temporomandibular joint pain: A double-blind and placebocontrolled trial," Oral Surgery, Oral Medicine, Oral Pathology, Oral Radiology, and Endodontology, vol. 105, no. 4, pp. 452-456, 2008.

[8] S. Kulekcioglu, K. Sivrioglu, O. Ozcan, and M. Parlak, "Effectiveness of low-level laser therapy in temporomandibular disorder," Scandinavian Journal of Rheumatology, vol. 32, no. 2 , pp. 114-118, 2003.

[9] E. M. Kogawa, M. T. Kato, C. N. Santos, and P. C. R. Conti, "Evaluation of the efficacy of low-level laser therapy (LLLT) and the microelectric neurostimulation (MENS) in the treatment of myogenic temporomandibular disorders: A randomized clinical trial," Journal of Applied Oral Science, vol. 13, no. 3, pp. 280-285, 2005.

[10] R. Abreu Venancio, C. M. Camparis, and R. Fatima Zanirato Lizarelli, "Low intensity laser therapy in the treatment of temporomandibular disorders: A double-blind study," Journal of Oral Rehabilitation, vol. 32, no. 11, pp. 800-807, 2005.

[11] S. C. Núñez, A. S. Garcez, S. S. Suzuki, and M. S. Ribeiro, "Management of mouth opening in patients with temporomandibular disorders through low-level laser therapy and transcutaneous electrical neural stimulation," Photomedicine and Laser Surgery, vol. 24, no. 1, pp. 45-49, 2006.

[12] L. A. Cunha, L. M. Firoozmand, A. P. Silva, S. A. Esteves, and W. Oliveira, "Efficacy of low-level laser therapy in the treatment of temporomandibular disorder," International Dental Journal, vol. 58, no. 4, pp. 213-217, 2008.

[13] T. Graciele Carrasco, M. Oliveira Mazzetto, R. Galli Mazzetto, and W. Mestriner, "Low intensity laser therapy in temporomandibular disorder: A phase II double-blind study," Cranio ${ }^{\circledR}$, vol. 26, no. 4, pp. 274-281, 2008.

[14] E. Lassemi, "Low-level laser therapy in the management of temporamandibular joint disorder," Journal of Oral Laser Applications, vol. 8, no. 2, 2008.

[15] M. O. Mazzetto, T. H. Hotta, and R. C. d. A. Pizzo, "Measurements of jaw movements and TMJ pain intensity in patients treated with GaAlAs laser," Brazilian Dental Journal, vol. 21, no. 4, pp. 356-360, 2010.

[16] T. Dostalová, P. Hlinakova, M. Kasparova, A. Rehacek, L. Vavrickova, and L. Navrátil, "Effectiveness of physiotherapy and GaAlAs laser in the management of temporomandibular joint disorders," Photomedicine and Laser Surgery, vol. 30, no. 5, pp. 275-280, 2012.

[17] C. H. L. De Godoy, S. Paula da Costa, A. Deise Sales de et al., "Evaluation of effect of low-level laser therapy on adolescents with temporomandibular disorder: Study protocol for a randomized controlled trial," Trials, vol. 14, no. 1, p. 229, 2013.

[18] M. H. C. d. V. Catão, P. S. d. Oliveira, R. d. O. Costa, and V. S. M. Carneiro, "Avaliação da eficácia do laser de baixa intensidade no tratamento das disfunções têmporo-mandibular: estudo clínico randomizado," Revista CEFAC, vol. 15, no. 6, pp. 1601-1608, 2013.

[19] A. S. Madani, F. Ahrari, F. Nasiri, M. Abtahi, and J. Tunér, "Low-level laser therapy for management of TMJ osteoarthritis," Cranio ${ }^{\circledR}$, vol. 32, no. 1, pp. 38-44, 2014.

[20] T. S. Pereira, O. D. Flecha, R. C. Guimarães et al., "Efficacy of red and infrared lasers in treatment of temporomandibular disorders - a double-blind, randomized, parallel clinical trial," Cranio $^{\circledR}$, vol. 32, no. 1, pp. 51-56, 2014. 
[21] N. Sayed, C. Murugavel, and A. Gnanam, "Management of temporomandibular disorders with low level laser therapy," Journal of Maxillofacial and Oral Surgery, vol. 13, no. 4, pp. 444-450, 2014.

[22] Y.-F. Huang, J.-C. Lin, H.-W. Yang, Y.-H. Lee, and C.-H. Yu, "Clinical effectiveness of laser acupuncture in the treatment of temporomandibular joint disorder," Journal of the Formosan Medical Association, vol. 113, no. 8, pp. 535-539, 2014.

[23] W.-L. Hu, C.-H. Chang, and Y.-C. Hung, "Laser acupuncture therapy in patients with treatment-resistant temporomandibular disorders," PLoS One, vol. 9, no. 10, Article ID e110528, 2014.

[24] M. Seifi, A. Ebadifar, S. Kabiri, M. R. Badiee, Z. Abdolazimi, and P. Amdjadi, "Comparative effectiveness of low level laser therapy and transcutaneous electric nerve stimulation on temporomandibular joint disorders," Journal of Lasers in Medical Sciences, vol. 8, no. 1, pp. S27-S31, 2017.

[25] F. Rezazadeh, K Hajian, S Shahidi, and S Piroozi, "Comparison of the effects of transcutaneous electrical nerve stimulation and low-level laser therapy on drug-resistant temporomandibular disorders," Journal of Dentistry, vol. 18, no. 3, p. 187, 2017.

[26] D. W. Douglas de Oliveira, F. S. Lages, R. C. Guimarães et al., "Do TMJ symptoms improve and last across time after treatment with red $(660 \mathrm{~nm})$ and infrared $(790 \mathrm{~nm})$ low level laser treatment (LLLT)? A survival analysis," Cranio ${ }^{\circledR}$, vol. 35, no. 6, pp. 372-378, 2017.

[27] M. Basili, "Low-level laser therapy in the treatment of muscleskelet pain in patients affected by temporomandibular disorders," Oral \& Implantology, vol. 10, no. 4, p. 406, 2017.

[28] C. H. L. de Godoy, L. J. Motta, E. J. Garcia et al., "Electromyographic evaluation of a low-level laser protocol for the treatment of temporomandibular disorder: A randomized, controlled, blind trial," Journal of Physical Therapy Science, vol. 29, no. 12, pp. 2107-2111, 2017.

[29] R. Shobha, V. Narayanan, B. S. Jagadish Pai, H. Jaishankar, and M. Jijin, "Low-level laser therapy: A novel therapeutic approach to temporomandibular disorder - A randomized, double-blinded, placebo-controlled trial," Indian Journal of Dental Research, vol. 28, no. 4, p. 380, 2017.

[30] S. Buduru, D. Talmaceanu, O. Baru, C. Culcitchi, and R. Cosgarea, "Low-level LASER therapy effects vs. placebo in the treatment of temporo-mandibular joint disorders," Balneo Research Journal, vol. 9, no. 3, pp. 281-284, 2018.

[31] A. Peimani, S. Keshavarz, and M. Fathollahi, "Comparison of low-level laser therapy and drug therapy in patients with temporomandibular disorders: A randomized clinical trial," Journal of Oral Health \& Dentistry, vol. 2, p. 205, 2018.

[32] A. Del Vecchio, M. Floravanti, A. Boccassini et al., "Evaluation of the efficacy of a new low-level laser therapy home protocol in the treatment of temporomandibular joint disorder-related pain: A randomized, double-blind, placebo-controlled clinical trial," Cranio ${ }^{\circledR}$, vol. 19, pp. 1-10, 2019.

[33] S. A. C. Tortelli, L. Saraiva, and D. C. Miyagaki, "Effectiveness of acupuncture, ozonio therapy and low-intensity laser in the treatment of temporomandibular dysfunction of muscle origin: A randomized controlled trial," Revista de Odontologia da UNESP, vol. 48, 2019.

[34] S. Khairnar, K. Bhate, S. K. Santhosh Kumar, K. Kshirsagar, B. Jagtap, and P. Kakodkar, "Comparative evaluation of lowlevel laser therapy and ultrasound heat therapy in reducing temporomandibular joint disorder pain," Journal of Dental Anesthesia and Pain Medicine, vol. 19, no. 5, pp. 289-294, 2019.
[35] F. E. Yamaner, T. Celakil, and B. Gökcen Roehlig, "Comparison of the efficiency of two alternative therapies for the management of temporomandibular disorders," Cranio ${ }^{\circledR}$, pp. 1-10, 2020.

[36] L. McGuinness, Robvis: An R Package and Web Application for Visualising Risk-of-Bias Assessments, National Institute for Health Research (NIHR), London, Uk, 2019. 